\title{
The Necessary Parameters Calculation for CSMC Mechanical Analysis
}

\author{
Xianewei Wang ${ }^{1}$, Zhaoliang Wang ${ }^{2}$, Qing $\mathrm{He}^{1}$, Xiulian $\mathrm{Li}^{1}$ \\ ${ }^{1}$ Jiangsu University of Technology, Changzhou 213001, China. E-mail: wangxw@jsut.edu.cn, qqaa611@163.com, \\ 936303415@qq.com \\ ${ }^{2}$ Institute of Plasma Physics, Chinese Academy of Sciences, Hefei 230031, China. E-mail: zhlwang@ipp.ac.cn
}

\begin{abstract}
The Central Solenoid Model Coil is a hybrid superconducting coil being developed in Institute of Plasma Physics Chinese Academy of Sciences. The R\&D of Central Solenoid Model Coil will lay the foundation for the fabrication of China Fusion Engineering Test Reactor Central Solenoid coil. One of the main purposes during the R\&D of Central Solenoid Model Coil is to verify its mechanical performance under the complicated load cases. In order to efficiently and accurately carry out the mechanical analysis, some necessary parameters such as the electromagnetic state under assembly error, the thermal contact resistance and the equivalent material property of conductor winding packet should be calculated in advance. The electromagnetic state here mainly refers to the asymmetric magnetic field and electromagnetic force. The calculation of magnetic density is based on elliptical integrals, the results will provide guidance for the assembly optimization of coil modules. And the subsequently obtained electromagnetic force will offer load boundary condition for the coil stability analysis. The thermal contact resistance is calculated under the assumption that the interface asperity approximately obeys the Gaussian distribution. The thermal contact resistance will be used in the thermal analysis. And the key advantage is which makes the prediction of preload force variation more accurate during coil the cooling down process. The equivalent material property is calculated by using generalized Hook's law and finite element method. Based on the equivalent material property, the coupling analysis model of CSMC will be significantly simplified. Moreover, it makes CSMC full model analysis under non-uniform loads become possible.
\end{abstract}

Keywords: CSMC, assembly error, magnetic density, equivalent material property, TCR

\section{Introduction}

Tokamak is a kind of magnetic confinement fusion device developed by human beings to explore the fusion energy. As one of the key components of Tokamak [1, 2], the Central Solenoid (CS) coil has the function of exciting the initial plasma current and maintaining the plasma shape. The Central Solenoid Model Coil (CSMC) is a hybrid superconducting coil being developed towards the China Fusion Engineering Test Reactor (CFETR) [3, 4, 5]. For the sake of saving space for the tritium breeding blanket and also decreasing the built cost, the superconducting materials of $\mathrm{Nb} 3 \mathrm{Sn}$ and $\mathrm{NbTi}$ are used together for CSMC. The coil modules winded by these two superconducting cables are located in the high and low magnetic field regions respectively. The peak magnetic field of CSMC is $12 \mathrm{~T}$ and the maximum magnetic field variation ratio is $1.5 \mathrm{~T} \cdot \mathrm{s}^{-1}[6]$. In order to ensure the integrity of CSMC after being energized, the preload force is loaded through the preload structure arranged along the circumferential direction, as shown in Fig.1.

One of the main tasks during the R\&D of CSMC is to study its superconducting characteristics under the complexed structural-electromagnetic-thermal loads. While it is not easy to directly launch the mechanical analysis. Since the coil winding packet is winded through the tiny size multistage cables and which finally form the extremely huge and complex structure, namely it is nearly impossible to create the refined model and carry out the related analysis by using a general workstation. However, once some key parameters are calculated in advance, they can be inputted as external parameters to simplify the full model and perform all kinds of mechanical analysis. The calculation method such as the equivalent material property of winding packet, the thermal contact resistance and the coil electromagnetic state under assembly error are presented in the paper.

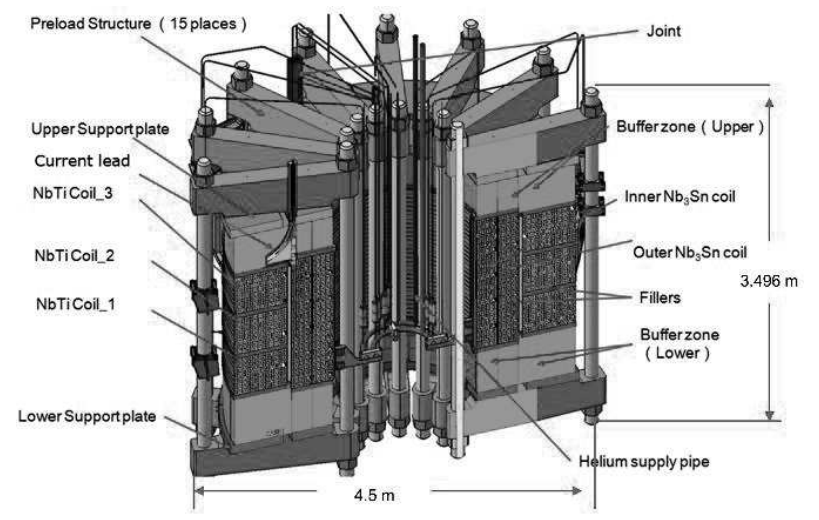

Fig. 1 The geometric structure of CSMC

\section{The magnetic field and electromagnetic force under assembly error}

CSMC is composed of five coil modules, namely the inner $\mathrm{Nb} 3 \mathrm{Sn}$ coil, outer $\mathrm{Nb} 3 \mathrm{Sn}$ coil, top $\mathrm{NbTi}$ coil, middle $\mathrm{NbTi}$ coil and bottom NbTi coil as shown in Fig. 2. The coil modules are axial symmetry with respect to axis $\mathrm{Z}$ and also symmetric about the middle plan RZ (equatorial plane). In view of the geometric symmetry, once CSMC is energized, the magnetic field induced by the pulse current will be uniform around the coil toroidal direction. The radial electromagnetic force caused due to the interaction of current and magnetic field is also uniform along the toroidal direction and the axial resultant force is zero. 
The current electromagnetic analysis is usually based on the above ideal state. While there is assembly clearance among the coil modules. The radial clearance between the inner and outer $\mathrm{Nb} 3 \mathrm{Sn}$ coil is $10 \mathrm{~mm}$. The radial clearance between the outer $\mathrm{Nb} 3 \mathrm{Sn}$ coil and $\mathrm{NbTi}$ coil is $50 \mathrm{~mm}$ and there are also $50 \mathrm{~mm}$ axial clearance among the NbTi coil modules. Given the current assembly technology, it is impossible to perfectly achieve the assembling without error. Corresponding to the assemble clearance, the assembly error contains the two different types namely the radial and axial errors, as shown in Fig. 3. Once the assembly error is introduced in the electromagnetic analysis, it becomes rather difficult to calculate the coil electromagnetic state by using finite element method because of the huge complicated finite element model. Where a simple method is given and the results can be used as the external load boundary for the following coupling analysis.

According to other tokamak CSMC assembly experience, assuming the radial and axial assembly errors between $\mathrm{Nb} 3 \mathrm{Sn}$ and $\mathrm{NbTi}$ coil are in the range of $[0,10$ $\mathrm{mm}]$. The coil magnetic density under assembly error is calculated by dividing the coil into 480 turns equivalent circular current carrying filament and the magnetic density caused by the filament is analysed based on elliptical integral $[7,8,9]$. The magnetic density on the innermost near to middle plane cable of inner Nb3Sn coil is presented in Fig. 4. The result indicates the magnetic density along the toroidal direction are not uniform anymore and the maximum variation of it is about $0.05 \mathrm{~T}$. The average unevenness is about $0.43 \%$. The calculation of electromagnetic force is based on Ampere's Force Law. The variation of radial and axial electromagnetic force versus the radial and axial assembly errors are shown in Fig. 5 and 6 respectively. It seems that the electromagnetic force ascends in the linear proportion about the assembly error and the axial electromagnetic force is about two times of the radial electromagnetic force under the same assembly error. The result can be used to estimate the electromagnetic force under the actual assembly error. In addition, since the electromagnetic force connected with the axial assembly error is larger than that related to the radial assembly error. The axial installation accuracy has the privilege to be assured primarily during the installation process.

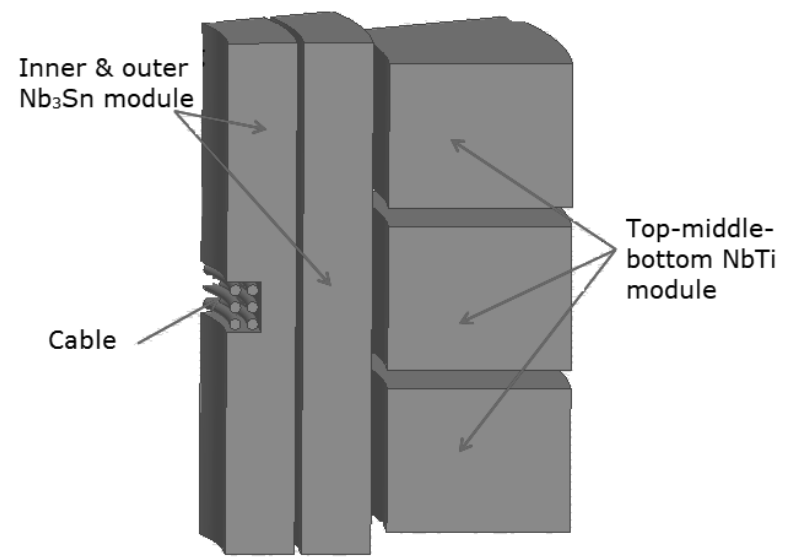

Fig. 2 The ideal coil modules without installation error

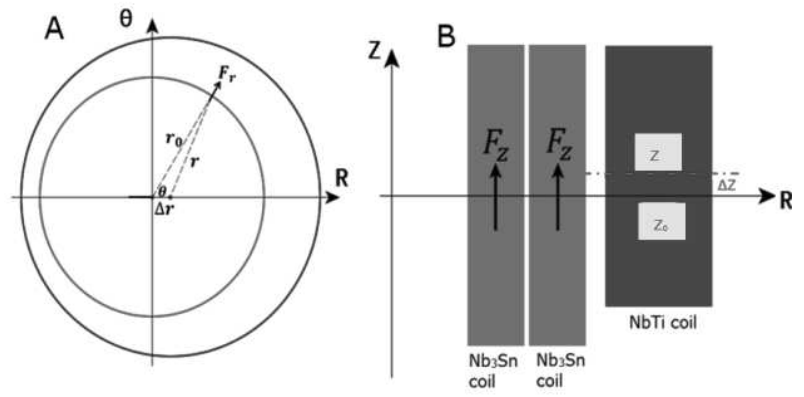

Fig. 3 (A) The radial misalignment of NbTi coil. (B) The axial misalignment of $\mathrm{NbTi}$ coil

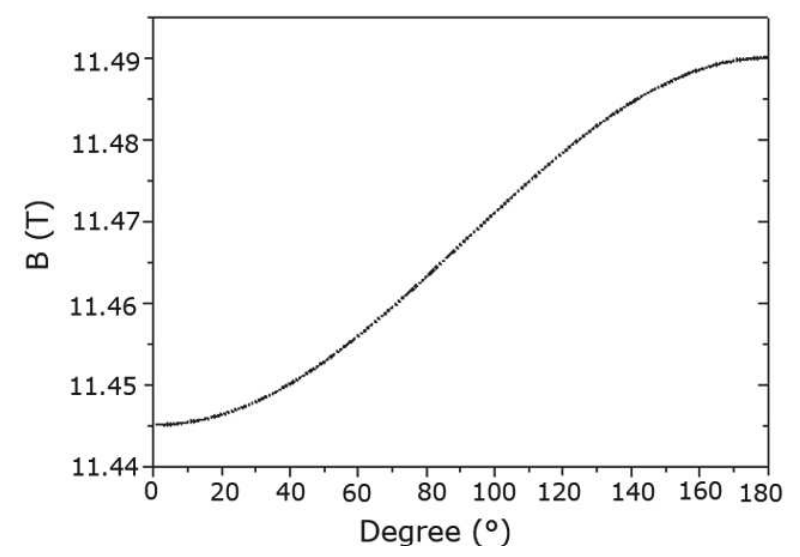

Fig. 4 The toroidal magnetic field of inner Nb3Sn coil innermost-near middle plane cable

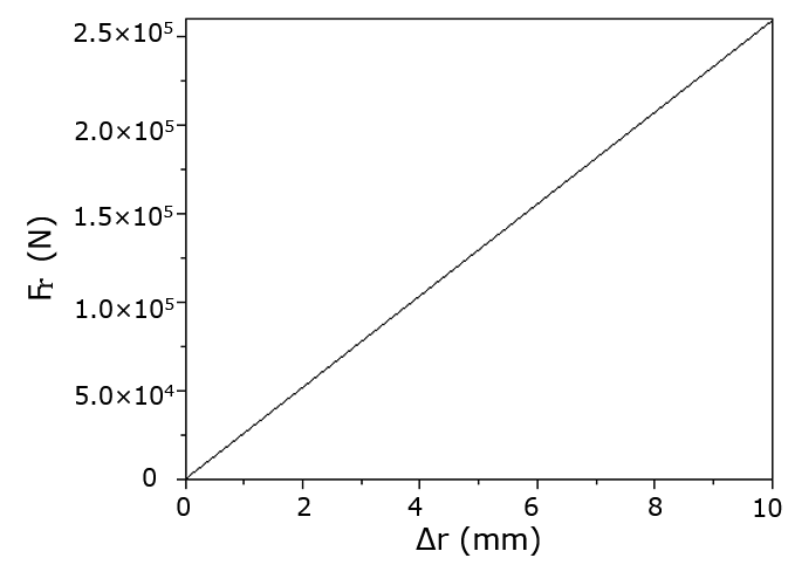

Fig. 5 The radial electromagnetic force of inner Nb3Sn coil under different radial misalignment

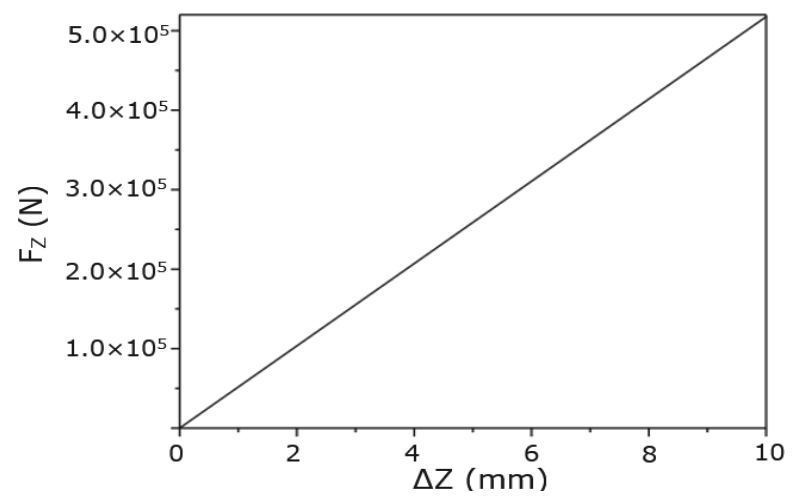

Fig. 6 The axial electromagnetic force of inner Nb3Sn coil under different axial misalignment 


\section{The TCR on component contact interface}

Before CSMC is energized, it should be cooled down from room temperature to $4.5 \mathrm{~K}$ by using the liquid nitrogen and helium. The temperature on the coil components is affected by several factors such as the cooling speed, Coefficient of material Thermal Expansion (CTE) and Thermal Contact Resistance (TCR). To avoid the large temperature gradient resulted from cooling speed, the cooling process is strictly controlled and it lasts about three weeks. The effect of CTE can be mitigated by adjusting the material combining scheme $[10,11]$, which mainly refers to the selection of insulation material and stainless steel. While the TCR will go through the overall process of heat transfer and it can be hardly eliminated. To accurately simulate the temperature distribution during the thermal analysis, the TCR should be calculated beforehand. Where the components of buffer zone and compression plate are selected to analyse the TCR, the other components can be calculated using the same method.

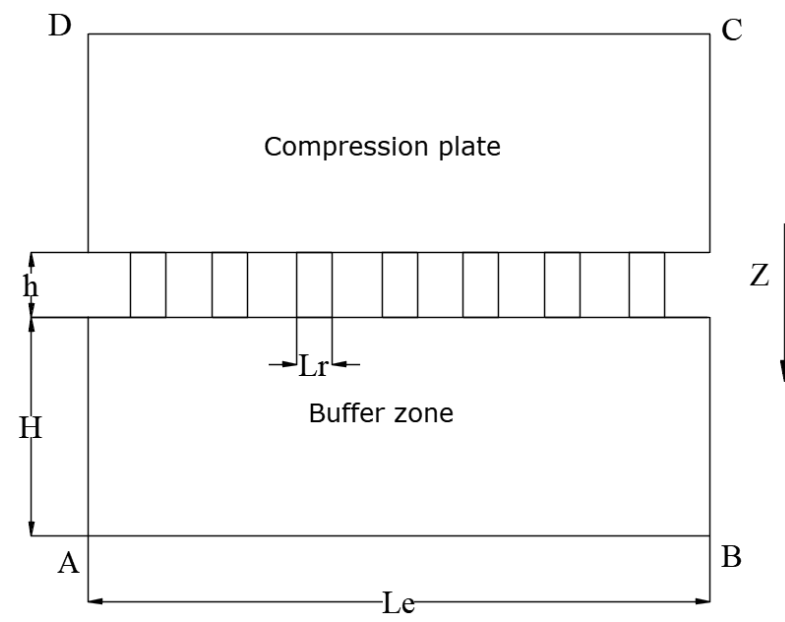

Fig.7 The calculation model of thermal contact resistence

For the specific calculation of TCR, assuming the interface asperity approximately obey the Gaussian distribution and the asperity shape is cylindrical $[12,13]$. The surfaces of load beam and the compression plate are equivalent to a rigid smooth surface and a random rough surface respectively. The asperity size and the contact gap ratio are experimentally measured and the average value are used in the calculation model, as shown in Fig. 7. Considering the allowable maximum temperature gradient between CSMC components is $50 \mathrm{~K}$, which is used in the conservative temperature boundary during the calculation of TCR. Where the temperature of $5 \mathrm{~K}$ and $55 \mathrm{~K}$ are applied to the external surface of buffer zone and compression plate respectively. The detailed temperature and heat flux at the interface are obtained by using the commercial software ANSYS as shown in Fig. 8. Then the TCR is calculated according to the equation (1). The TCR under different temperature is shown in Fig. 9. The conspicuous variation of TCR occurs when CSMC is cooled down to operation temperature.

$$
R=\frac{\frac{1}{n} \sum_{i=1}^{n} T_{h i}-\frac{1}{m} \sum_{i=1}^{m} T_{l i}}{\left(\frac{1}{n} \sum_{i=1}^{n} q_{h i}+\frac{1}{m} \sum_{i=1}^{m} q_{l i}\right) / 2}\left[\mathrm{~m}^{2} \mathrm{KW}^{-1}\right],
$$

Where:

$n$ Number of points extracted on the high temperature side [-],

$m$ Number of points extracted on the low temperature side [-],

$T_{h i} \quad$ The temperature of point $i$ in the high temperature side $[\mathrm{K}]$,

$T_{l i} \quad$ The temperature of point $i$ in the low temperature side $[\mathrm{K}]$,

$q_{h i}$ The heat flux of point $i$ in the high temperature side $\left[\mathrm{Wm}^{-2}\right]$,

$q_{l i}$ The heat flux of point $i$ in the low temperature side $\left[\mathrm{Wm}^{-2}\right]$.

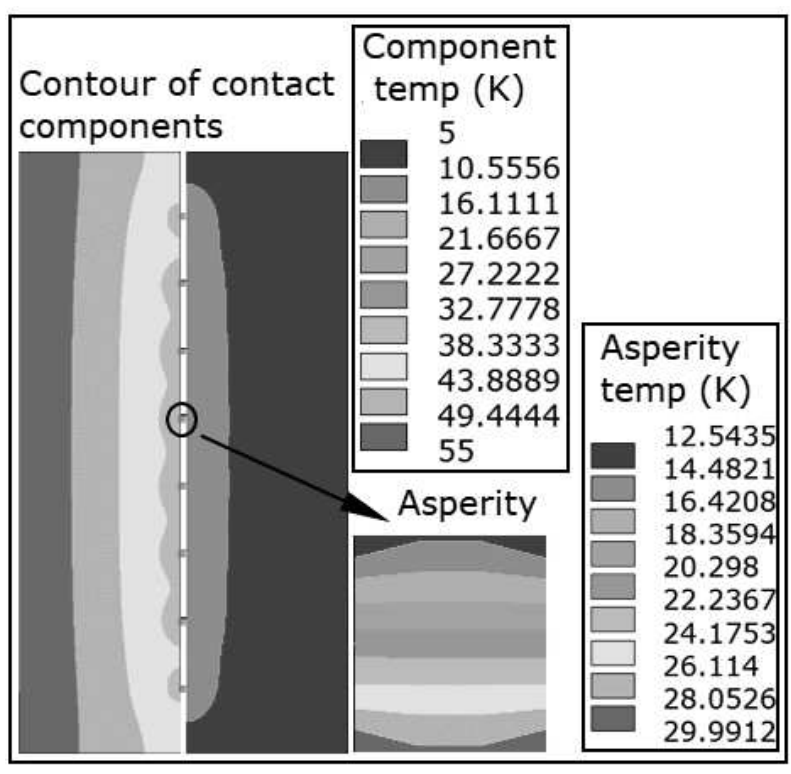

Fig.8 Temperature distribution on $316 L N$ and G10 test samples

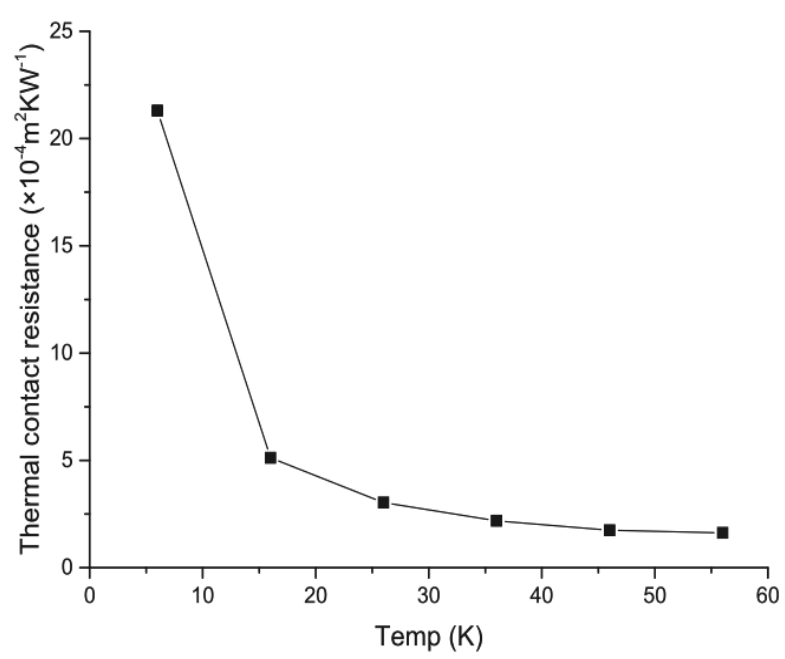

Fig. 9 The variation of thermal contact resistance with temperature 


\section{The calculation of equivalent material prope- rties}

CSMC winding packet is winded by the Cable In Conduit Conductor (CICC) as shown in Fig. 10. We only present the equivalent material properties of $\mathrm{Nb} 3 \mathrm{Sn}$ winding packet in the paper. The equivalent material properties of $\mathrm{NbTi}$ winding packet can be calculated by using the same method. Because of the huge geometric size of CSMC, it is difficult to build the detail winding packet model in the mechanical analysis. Although the one fifteenth model containing CICC is built in reference [14] by using computer cluster, the model is not suitable to be used for the stability and seismic analysis. To avoid the expensive calculation cost, a simplified model with the equivalent material properties is proposed here. Now that the coil winding packet is periodically stacked by the fine-scale unit cell, which is used as the analysis object to calculate the equivalent material properties of winding packet. Each unit cell is made up of the inner superconducting cable, middle stainless steel, and outer insulator. The superconducting cable with voids is omitted in the calculation due to its small rigidity. The final calculation models are shown in Fig. 11, which include two different arrangement form namely the aligned \& misaligned. The numerical solution is carried out based on homogenization theory by using finite element method $[15,16,17]$. According to the generalized Hooke's law, the equivalent elastic modulus and Poisson's ratio are calculated as per the equations (2)-(6).

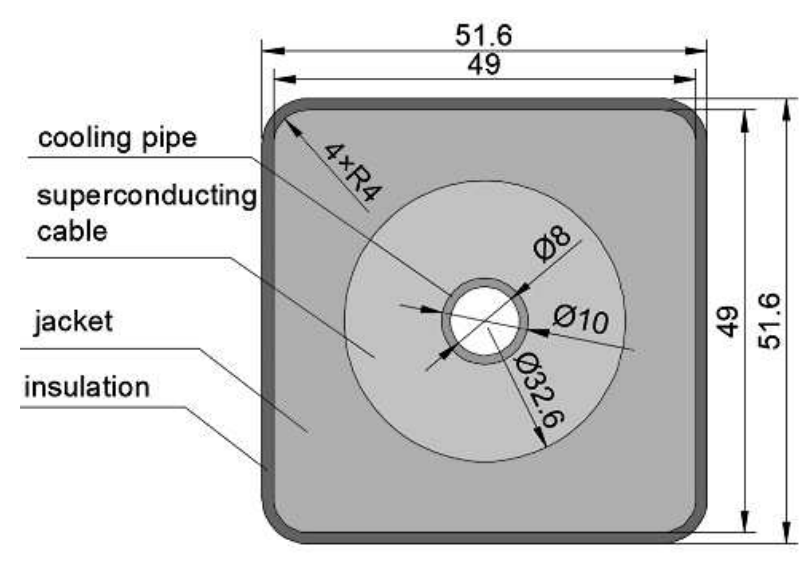

Fig. 10 The geometric structure of Nb3Sn CICC

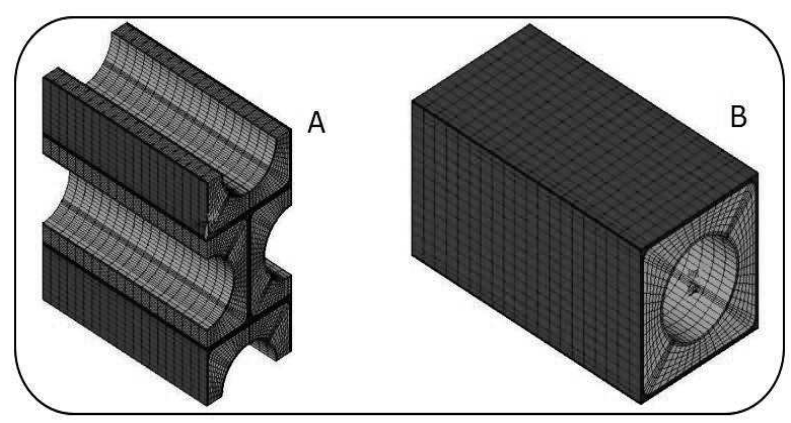

Fig. 11 The aligned \& misaligned unit cell. (A) Misalig-

$$
\begin{aligned}
& \text { ned. (B) Aligned } \\
& \varepsilon=D^{-1} \sigma[-],
\end{aligned}
$$

Where:

$D$ Rigidity coefficient [-],

$\sigma$ Stress in the material $[\mathrm{Pa}]$.

$$
D^{-1}=\left[\begin{array}{cccccc}
1 / E_{x} & -\nu_{x y} / E_{x} & -\nu_{x z} / E_{x} & 0 & 0 & 0 \\
-\nu_{y x} / E_{y} & 1 / E_{y} & -\nu_{y z} / E_{y} & 0 & 0 & 0 \\
-\nu_{z x} / E_{z} & -\nu_{z y} / E_{z} & 1 / E_{z} & 0 & 0 & 0 \\
0 & 0 & 0 & 1 / G_{x y} & 0 & 0 \\
0 & 0 & 0 & 0 & 1 / G_{y z} & 0 \\
0 & 0 & 0 & 0 & 0 & 1 / G_{z x}
\end{array}\right][-]
$$

Where:

$E_{x}, E_{y}, E_{z} \quad$ Elastic modulus in the $x, y, z$ directions respectively $[\mathrm{Pa}]$,

$G_{x y}, G_{y z}, G_{z x}$ Shear modulus in the $x y, y z, z x$ planes respectively $[\mathrm{Pa}]$,

$E_{x}, E_{y}, E_{z} \quad$ Elastic modulus in the $x, y, z$ directions respectively $[\mathrm{Pa}]$,

$v_{x y}, v_{y z}, v_{z x} \quad$ Possion's ratio corresponding to $x-y, y-z, z-x$ respectively [-].

$\varepsilon_{x}=\overline{U_{x}} / L_{x}, \varepsilon_{y}=\overline{U_{y}} / L_{y}, \varepsilon_{z}=\overline{U_{z}} / L_{z}[-]$,

$\sigma_{x}=-P_{x}, \sigma_{y}=0, \sigma_{z}=0[\mathrm{~Pa}]$,

$E_{x}=\sigma_{x} / \varepsilon_{x}, \nu_{x y}=-\varepsilon_{y} / \varepsilon_{x}, \nu_{x z}=-\varepsilon_{z} / \varepsilon_{x}[\mathrm{~Pa}],[-],[-],(6)$

Where:

$\varepsilon_{x}, \varepsilon_{y}, \varepsilon_{z} \quad$ Elastic strain in the $x, y, z$ directions respectively [-],

$\bar{U}_{x}, \bar{U}_{y}, \bar{U}_{z} \quad$ Material average deformation in the $x, y, z$ direction respectively [m],

$L_{x}, L_{y}, L_{z} \quad$ The length of the calculation model in the $x, y, z$ direction respectively $[\mathrm{m}]$,

$\sigma_{x}, \sigma_{y}, \sigma_{z}$ Stress in the $x, y, z$ direction respectively $[\mathrm{Pa}]$,

$P_{x}$ the normal pressure applied on the model $x$ direction $[\mathrm{Pa}]$.

The shear modulus can be calculated as per the equations (7)-(9) by applying shear force on the model. 


$$
\begin{aligned}
& \tau_{y z}=\Sigma F_{z} / A_{x z}, \gamma_{y z}=U Z_{b} / L_{y}, G_{y z}=\tau_{y z} / \gamma_{y z}[\mathrm{~Pa}],[-],[\mathrm{Pa}], \\
& \tau_{y z}=\Sigma F_{z} / A_{x z}, \gamma_{x y}=U X_{b} / L_{y}, G_{x z}=\tau_{y z} / \gamma_{x y}[\mathrm{~Pa}],[-],[\mathrm{Pa}], \\
& \tau_{x z}=\Sigma F_{y} / A_{y z}, \gamma_{x z}-U Z_{b} / L_{x}, G_{y z}-\tau_{x z} / \gamma_{x z}[\mathrm{~Pa}],[-],[\mathrm{Pa}],
\end{aligned}
$$

Where:

$\tau_{y z}, \tau_{z x}$ Shear stress in the $y z, z x$ planes respectively $[\mathrm{Pa}]$,

$\Sigma F_{y}, \Sigma F_{z} \quad$ Applied sum shear force on the model

$y, z$ direction respectively [N],

$A_{x z}, A_{y z} \quad$ Model surface areas of area $x-z, y-z$ respectively $\left[\mathrm{mm}^{2}\right]$,

$U$ The total deformation under the shear force $[\mathrm{mm}]$,

$X_{b}, Z_{b}$ Coordinate value of end point $\mathrm{b}[\mathrm{mm}]$,

$\gamma_{x y}, \gamma_{y z}, \gamma_{z x}$ Shear strain in the $x y, y z, z x$ planes respectively [-].

The material deformation involved in equations (4), (7), (8) and (9) are calculated through ANSYS simulation, the results are shown in Fig. 12 and 13. The eventually obtained equivalent material properties are listed in table 2 . Note that the variable subscript ${ }^{x}$ represent the direction through the insulator thickness, and ${ }^{y, z}$ are along the insulator layer direction.
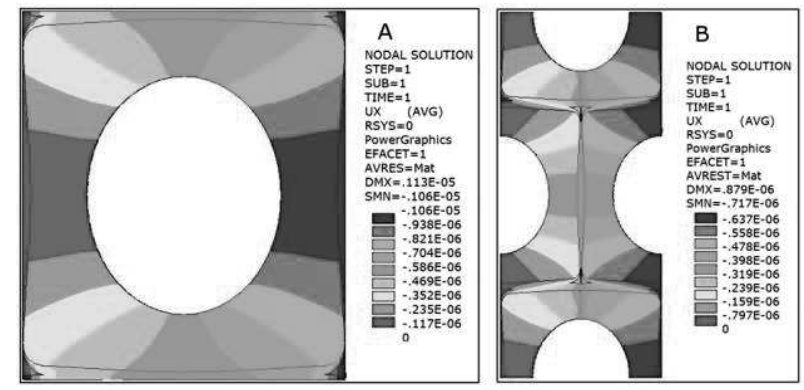

Fig. 12 Unit cell deformation in $x$ direction corresponding to elastic modulus calculation under aligned(A) and misaligned (B)
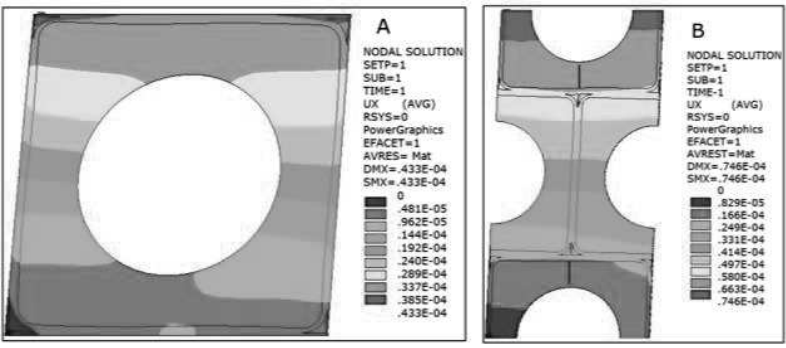

Fig. 13 Unit cell deformation in $x$ direction corresponding to shear modulus calculation under aligned(A) and misaligned (B)

Tab. 2 The equivalent material properties of Nb3Sn winding packet

\begin{tabular}{cccccccccc}
\hline $\begin{array}{c}\text { Arrangement } \\
\text { types }\end{array}$ & $\mathrm{E}_{x}(\mathrm{GPa})$ & $\mathrm{E}_{y}(\mathrm{GPa})$ & $\mathrm{E}_{z}(\mathrm{GPa})$ & $G_{x y}(\mathrm{GPa})$ & $G_{y z}(\mathrm{GPa})$ & $G_{z x}(\mathrm{GPa})$ & $N U_{x y}$ & $N U_{y z}$ & $N U_{z x}$ \\
\hline aligned & 48.9 & 46.6 & 120.4 & 6.43 & 19.9 & 17.6 & 0.3 & 0.12 & 0.12 \\
misaligned & 49.3 & 44.8 & 120.25 & 9.32 & 14.5 & 13.8 & 0.3 & 0.11 & 0.11 \\
\hline
\end{tabular}

\section{Conclusion}

CSMC is a very huge and complicated superconducting coil. In order to effectively and accurately evaluate the coil mechanical characteristics at some specific load cases, the conductor electromagnetic performance under assembly error, the component interface thermal contact resistance and winding packet equivalent material properties are calculated respectively. The electromagnetic analysis results indicate that the conductor peak magnetic field is less than $12 \mathrm{~T}$ under the assembly error. The unevenness of magnetic field along the circumferential direction is no more than $0.5 \%$. While the induced electromagnetic force under the $10 \mathrm{~mm}$ misalignment reach up to several times of the coil dead weight, which would be apt to influence the stability of the coil. Moreover, the electromagnetic force is in linear relation with assembly error, which provide the convenience to quickly predict the electromagnetic load at different assembly error. Given the super lower operation temperature, it is quite difficult to properly measure the component interface thermal contact resistance. The simulation method presented in the paper could quickly predict the thermal con- tact resistance. And it has the advantage to more accurately carry out the thermal analysis with the TRC obtained. The mechanical analysis model will be significantly simplified by using the equivalent material property. The equivalent calculation method used for Nb3Sn winding packet is also applicable to the analysis of NbTi winding packet. And it makes the full model analysis be achievable in some non-uniform load cases by using the equivalent material property.

\section{Acknowledgment}

This project has been financially supported by the $\mathrm{Na}$ tural Science Foundation of China (Grant No. 51605208, 51805230).

\section{References}

[1] YANG X J, LI L L (2016). Magneto-inertial fusion: A new approach towards fusion energy[J]. Scientia Sinica Physica, Mechanica \& Astronomica, Vol. 46, No. 11, pp. 115202. Scichina. China. 
[2] J. ONGENA, R. KOCH, R.WOLF, et al (2016). Magnetic Confinement Fusion[J]. Nature Physics, Vol. 12, No. 7, pp. 398-410. Nature publishing group. England.

[3] YU WU, YI SHI, JIANGANG LI, et al (2018). Basic design and progress of central solenoid model coil for CFETR[J]. IEEE Transactions on applied superconductivity, Vol. 28, No. 3, pp. 4200205. IEEE-inst electrical electronics engineers. USA.

[4] YUANXI WAN, JIANGANG LI, YONG LIU, et al (2017). Overview of the present progress and activities on the CFETR[J]. Nuclear Fusion, Vol. 57, No. 10, pp. 102009. IOP. England.

[5] HAN H X, WU Y, QIN J G, et al (2018). Winding R\&D for CFETR Central Solenoid Model Coil[J]. IEEE Transactions on applied superconductivity, Vol. 28, No. 3, pp. 4201905. IEEE-inst electrical electronics engineers. USA.

[6] SHI Y, WU Y, HAO QW, et al (2016). The AC loss evaluation of central solenoid model coil for CFETR[J]. Fusion Engineering and Design, Vol. 107, pp. 100-107, Elsevier. Switzerland.

[7] YUESEN CHU (1999). Numerical calculation for the Magnetic field in current-carrying circular arc filament[J]. IEEE Transactions on magnetics, Vol.27, No. 2, pp.1588-1595. IEEE-inst electrical electronics engineers. USA.

[8] LAXMIKANTK, URANKAR (1980). Vector potential and magnetic field of current-carrying finite arc segment in analytical form, part 1: filament approximation[J], IEEE Transactions on magnetics, Vol.16, No. 5, pp.1283-1288. IEEEinst electrical electronics engineers. USA.

[9] XIANWEI WANG, ZHAOLIANG WANG, FEI XIE, et al (2019). Electromagnetic Analysis of the Updated Fast Control Coil for EAST[J]. Manufacturing Technology. Vol. 19, No. 1, pp. 172-176. Engineering Village. USA.
[10] H. TSUJI, S. EGOROV, J. MINERVINI et al (2001). ITER R\&D: Magnet: Central Solenoid Model Coil[J]. Fusion Engineering and Design, Vol. 55, pp. 153-170. Elsevier. Switzerland.

[11] N. MARTOVETSKY, P. MICHAEL, J. MINERVINI et al (2002). Test of the ITER Central Solenoid Model Coil and CS Insert[J]. IEEE Transaction on Applied Superconductivity, Vol. 12, No. 1, pp. 600-605. IEEE-inst electrical electronics engineers. USA.

[12] GREENWOOD JA, WILLIAMSON JB (1996). Contact of nominally flat surfaces. In: Proceedings of the Royal Society of London Series AMathematical and Physical Sciences, pp. 300-319. Royal Society, UK.

[13] GREENWOOD JA, TRIPP JH (1970-1971). The contact of two nominally flat rough surfaces. In: Proceeding of the Institution of Mechanical Engineers, pp. 625-633. Royal Society, UK.

[14] XIAOGANG LIU, ZHAOLIANG WANG, YONG REN, et al (2018). Mechanical performance evaluation of the CFETR central solenoid model coil design[J]. Nuclear Fusion, Vol. 58, No. 1, pp. 016035. IOP. England.

[15] WANG J Q, WU S T, SONG Y T, et al (2006). Prediction of equivalent modulus of superconducting busbar for ITER[J]. Nuclear Fusion Plasma Physics, Vol.26, No.2, pp.100-104. Atomic energy press. China.

[16] LIU W Y, WU W Y, ZENG W B, et al (2013). Finite Element Analysis of Equivalent Material Properties of ITER Correction Coils[J]. Atomic Energy Science Technology, Vol.44, No.6, pp.745-749. Atomic energy press. China.

[17] SONTAMINO, ARKARAPON, PHANITWONG, WIRIYAKORN. (2017). Finite element analysis of counterbore-shaped parts by using sheet-bulk metal forming process[J]. Manufacturing Technology, Vol. 17, No. 4, pp. 597-602. Engineering Village. USA. 